\title{
Intermédialités
}

Histoire et théorie des arts, des lettres et des techniques

Intermediality

History and Theory of the Arts, Literature and Technologies

\section{Raconter la performance : l'entretien comme cadre pour la reprise et la transmission des performances. Entretiens avec Esther Ferrer et Nil Yalter}

\section{Clélia Barbut}

Numéro 28-29, automne 2016, printemps 2017

refaire

redoing

URI : https://id.erudit.org/iderudit/1041081ar

DOI : https://doi.org/10.7202/1041081ar

Aller au sommaire du numéro

Éditeur(s)

Revue intermédialités (Presses de l’Université de Montréal)

ISSN

1920-3136 (numérique)

Découvrir la revue

Citer cet article

Barbut, C. (2016). Raconter la performance : l'entretien comme cadre pour la reprise et la transmission des performances. Entretiens avec Esther Ferrer et Nil Yalter. Intermédialités / Intermediality, (28-29).

https://doi.org/10.7202/1041081ar
Résumé de l'article

À partir d'entretiens avec les artistes contemporaines Esther Ferrer et Nil Yalter, l'auteure interroge les vertus de l'entretien pour l'histoire de l'art et en particulier pour l'histoire des oeuvres performatives. Elle aborde les enjeux de l'oralité comme source et comme matière, et tente d'ouvrir des pistes de recherche historiographiques autour des affects et des incertitudes. 


\title{
Raconter la performance : l'entretien comme cadre pour la reprise et la transmission des performances
}

\author{
Entretiens avec Esther Ferrer et Nil Yalter
}

\author{
Clélia Barbut
}

\section{INTRODUCTION}

et article interroge les vertus de l'entretien pour l'histoire de l'art contemporain et plus précisément pour l'histoire des performances. Je m'appuierai sur le contenu de deux entretiens réalisés en 2016 avec Esther Ferrer et Nil Yalter, portant sur leur travail pendant et depuis la décennie 1970. Les quarante et quelques années qui séparent nos conversations des œuvres commentées représentent plus d'une génération, et l'entretien est l'occasion de restituer non seulement des œuvres, mais aussi le contexte historique qui les a vues naître ainsi que leurs expériences subjectives dans ce contexte. Tel que nous l'envisagerons ici, il permet de croiser, autour d'un questionnement au sujet des performances et de la reprise, les problématiques liées aux archives orales de l'art contemporain avec celles des historiographies féministes.

$I_{2} \quad$ La performance pendant les années 1970 est souvent une pratique artistique alternative, minoritaire, peu visible, soutenant une forme d'engagement. Les deux artistes interrogées soulignent la difficulté supplémentaire des femmes en la matière et déplorent un décalage important entre le temps de la création et celui de la reconnaissance. L'enjeu du récit oral est de se remémorer et de sélectionner des souvenirs personnels afin de les transmettre; il vise à évoquer au présent et à mettre en paroles des actes passés. Il est d'autant plus important qu'il permet aussi de 
réintégrer les souvenirs personnels dans cette histoire que Georges Perec appelle « l'histoire avec sa grande hache ${ }^{\mathrm{I}} »$, celle qui découpe des récits officiels et trace des histoires institutionnelles, c'est-à-dire, pour l'histoire de l'art, celle des expositions, des collections, qui est aussi une histoire d'exclusions. Dans la continuité des travaux en histoire de l'art contemporain qui visent à dépasser l'« effacement » des artistes marginalisés des histoires dominantes, l'entretien au sujet de la performance apparaît donc d'abord comme un moyen pour réhabiliter certains des « corps perdus $^{2} \gg$. Cette expression est employée par Amelia Jones pour souligner qu'il y a un enjeu historiographique important à tracer et à retracer les histoires des performeuses et performeurs qui, à partir des décennies 1960 et 1970, se sont situés en périphérie des mondes de l'art légitime. La méthode de l'histoire orale présente la vertu d'avoir une proximité essentielle avec les objets d'étude minoritaires : pour la discipline qui l'intègre progressivement pendant les années 1970, l'oralité est d'abord en effet «l'espace de l'autre ${ }^{3} »$. Le croisement de cette méthode avec l'histoire de l'art de la performance prend donc son sens dans ce geste doublement tourné vers une écriture de l'altérité. Les entretiens d'artistes au sujet des pratiques performatives sont des sources précieuses ${ }^{4}$, dont l'intégration au champ des pratiques historiennes se traduit aussi aujourd'hui dans les institutions d'archives qui collectionnent et parfois même collectent ces entretiens's. Comme le soulignent

I Georges Perec, $W$ on le souvenir d'enfance [1975], Paris, Gallimard, coll. « L'imaginaire », 20I5, p. I7.

${ }^{2} \ll[\ldots]$ the emphasis in "lost bodies" is on the erasure of certain practices from a range of dominant histories, and of the not coincidental fact that most of these practices surfaced or activated bodies as a means of politicizing art, making bodies otherwise kept out of high cultural domains visible. » Amelia Jones, «Lost Bodies. Early 1970s Los Angeles Performance Art in Art History », dans Peggy Phelan (dir.), Live Art in L.A.: Performance in Southern California, 1970-1983, Abingdon, Routledge, 20I2, p. II5.

${ }^{3}$ Michel de Certeau, L'écriture de l'bistoire [1975], Paris, Gallimard, coll. «Folio Histoire », 2007, p. 245. Voir aussi Dominique Aron-Schnapper et Danièle Hanet, «Archives orales et histoire des institutions sociales », Revue française de sociologie, vol. 2, $\mathrm{n}^{\circ} 2,1978$, p. 26I-275.

4 Voir par exemple Linda M. Montano, Performance Artists Talking in the Eighties, Berkeley, University of California Press, 2000.

5 Le programme « Archives orales de l'art de la période contemporaine, 1950-2010 » de l'Institut national d'histoire de l'art à Paris en partenariat avec les Archives de la critique d'art de Rennes, https://www.inha.fr/fr/recherche/le-departement-des-etudes-et-de-larecherche/domaines-de-recherche/histoire-de-1-art-contemporain-xxe-xxie-siecles/archivesorales-de-l-art-de-la-periode-contemporaine.html; le volet d'entretiens réalisés et diffusés dans le cadre du programme « Pacific Standard Time: Art in L.A. Oral History Interviews with 
Medhi Brit et Sandrine Meats dans un recueil d'entretiens publié récemment autour de la performance :

De la recherche à l'écriture, l'entretien, sa mise en place et sa diffusion s'imposent comme une entreprise dont la rhétorique même interroge la place de la parole dans la transmission d'un contenu et d'une esthétique, ouvrant tout un champ de réflexion sur les outils méthodologiques propres à l'historien et au curateur' .

La réflexion ici proposée se situe dans ce « champ de réflexion », c'est-à-dire dans le sillage des recherches qui soulignent les enjeux historiographiques de l'échange oral et les qualités de la parole pour la transmission des ouvres contemporaines, en particulier performatives. Or, la performance étant souvent caractérisée par le peu de traces qu'elle laisse, elle est tout particulièrement sujette aux variations mémorielles; les problématiques liées à sa transmission doivent donc être pensées avec acuité et attention. Comme certains.ne.s commencent à le relever, l'histoire de l'art véhiculée par les sources orales est une histoire $d^{\prime}$ « inadéquations ${ }^{7} »:$ manquant de sources primaires, elle est produite par des mémoires subjectives et consignée dans des récits « situés ${ }^{8}$ ». Nous verrons en effet que les témoignages d'Esther Ferrer et de Nil Yalter, envisagés sous l'angle de la reprise, invitent à penser des principes narratifs articulés autour des affects et des

Artists, Filmmakers, Curators, Collectors, and Critics, 2008-2012 » du Getty Research Institute à Los Angeles, http://www.oac.cdlib.org/findaid/ark:/13030/ktizogrghi/admin/.

${ }^{6}$ Mehdi Brit et Sandrine Meats, Interviewer la performance, Paris, Manuella Éditions, 2014, p. 27.

7 En 2009, l'INHA a par exemple accueilli l'installation vidéographique de l'artiste Silvia Kolbowski, dans une exposition intitulée An Inadequate History of Conceptual Art. L'exposition est composée en partie de vidéos de personnes témoignant au sujet d'œuvres d'art conceptuel qui les ont marqués. Dans ces vidéos, l'image encadre avec un zoom rapproché les mains de la personne qui raconte : les doigts se croisent et se nouent, pianotent tandis que le récit se déploie à travers leurs sensations et leurs souvenirs marquants, ainsi que leurs nombreuses imprécisions. Johanne Lamoureux souligne que les différents enjeux de ce projet concernent : «La vision qui se dégage de l'articulation entre expérience et mémoire, entre mémoire et histoire, [...] la mise en garde qui s'y fait jour quant aux limites et aux lacunes des sources premières, et enfin les ambiguités ouvertes par le point de vue des acteurs dans la fabrication de l'histoire. » Johanne Lamoureux, «Introduction », dans Marine Acker (dir.), An Inadequate History of Conceptual Art, Paris, Institut national de l'histoire de l'art, 2016, p. 8.

${ }^{8}$ Comme le souligne également Johanne Lamoureux à propos de ce dispositif de narration, un récit situé est personnel, c'est-à-dire qu'il peut être sujet aux variations (incertitudes) et aux humeurs (affects). Ibid. 
incertitudes. Ma réflexion mettra l'accent sur l'aspect incarné et lacunaire de la mémoire vive, pour y déceler des pistes de réflexion historiographiques : il s'agit donc de continuer d'emprunter ces « voies de traverse 9 » qui caractérisent l'écriture historiographique et les modes de mémorisation propres aux arts performatifs.

À travers la restitution du contenu de ces deux entretiens, je propose non seulement de donner de la visibilité à des pratiques qui en ont (eu) trop peu dans l'histoire de l'art, mais aussi de questionner les types de subjectivation et de narration qui sont produits par leur précarité. Quelles sont les formes de souvenir et de mémoire qui circulent dans l'échange parlé ? Quels types de posture, d'attention et d'engagement ces témoignages requièrent-ils de la part des historiens.ne.s ? Il s'agira de prêter attention aux descriptions des actions proposées par Esther Ferrer et Nil Yalter, mais aussi de les analyser à partir de la matière même de l'oralité, c'est-àdire des modulations de la voix, des répétitions, des silences, des omissions. Le premier temps de ma réflexion s'intéresse aux œuvres, aux formes que le récit peut donner aux ouvres de performance et à l'appréhension que les historiens.ne.s peuvent en avoir. Le fait que le récit s'inscrive dans un temps commun à la narratrice et à la personne qui écoute implique un partage sensible qui affecte la matière même des œuvres transmises, qui sont déterminées par les sons et les émotions. Cette matérialité de l'oralité requiert une forme d'engagement empathique, une prise en compte de la subjectivité et de la vulnérabilité des sujets abordés. On verra dans un second temps que, parsemés d'oublis et d'hésitations, de doutes et d'anecdotes, les récits d'Esther Ferrer et de Nil Yalter amènent une précarité, narrative et économique, au cour de l'histoire de leurs œuvres performatives. On soulignera que les incertitudes et les lacunes propres à ces récits sont caractéristiques des formes narratives qui ont partie liée avec une perspective féministe et peuvent, dès lors, être pensées comme des matériaux historiographiques à part entière.

\section{LE RÉCIT ET LE RESSENTI : UNE HISTOIRE DES AFFECTS ET DES ÉMOTIONS}

La loi du récit oral, de l'anecdote au mythe, est également la circulation. Il s'agit soit d'écouter une histoire, puis d'en raconter une à son tour, soit de

9 Anne Bénichou(dir.), Recréer, scripter. Mémoire et transmission des auvres performatives contemporaines, Dijon, Les presses du réel, 2015, p. 258. 
raconter l'histoire entendue et de la colporter dans un autre groupe, une autre communauté. L'histoire s'échange non seulement contre une autre histoire, mais aussi contre de la reconnaissance, de l'amour, de la considération, etc. [...] Le récit est une économie en soi, mais il est en quelque sorte contre-productif, dans le sens où il ne produit pas de capital. Il a pour fonction de distraire, de susciter la curiosité ou le désirir ${ }^{\mathrm{IO}}$.

Dans une réflexion sur le rôle du récit oral dans le champ de l'art contemporain et plus particulièrement de l'anecdote, Claire de Ribaupierre le place ici dans une économie $\mathrm{du}$ don et $\mathrm{du}$ contre-don: le récit échangé participe d'une forme d'échange non marchand et « contre-productif », qui ne produit pas de plus-value. En effet, la plupart du temps, les entretiens ne sont pas rémunérés et encadrent une circulation exclusivement symbolique de la valeur entre le narrateur ou la narratrice et l'auditeur.trice; c'est le cas de ceux qui seront mentionnés. Les valeurs et significations qui peuvent circuler pendant un entretien (《reconnaissance », 《amour », « considération », « curiosité », « désir ») sont liées au registre affectif. Il y a bien, néanmoins, un échange de valeur entre les deux positions, car le travail du ou de la scientifique consiste précisément à raconter, à partir du récit de la personne qui témoigne, une «autre histoire» auprès d'une «autre communauté ». Dans le cas d'un entretien avec un.e artiste, la diffusion auprès de la communauté scientifique peut amener une forme de reconnaissance. Le récit des œuvres est inévitablement orienté par cette circulation potentielle: l'artiste peut choisir son degré d'implication dans la description, les énoncés sur lesquels elle ou il insiste $^{\text {II }}$. En face, la personne qui écoute doit être active et produire des signes de considération qui entretiennent la volonté de raconter.

Je montrerai à partir des témoignages d'Esther Ferrer et de Nil Yalter que l'entretien a des vertus spécifiques en ce qui concerne la transmission de la performance, car les ambiances sonores et les émotions que l'échange oral fait saillir sont précieuses, souvent essentielles pour saisir les ouvres performatives. Cette

\footnotetext{
Io Claire de Ribaupierre, Anecdote, Zürich, JRP/Ringier, 2007, p. 9.

II J'ai développé une réflexion à propos de l'usage de l'entretien pour produire ou rétablir les « vérités » historiques des performances : Clélia Barbut, « Du document aux entretiens. Transmission, mémoire et historiographie des performances féministes des années 1970 », dans Émilie Blanc, Marie-Laure Allain Bonilla, Johanna Renard et Elvan Zabunyan(dir.), Subjectivités féministes, queer et postcoloniales en art contemporain, Rennes, Presses Universitaires de Rennes, à parâtre.
} 
transmission par les sens et les sons implique une forme de coprésence sensible et physique, une attention réciproque qui peut être éclairée par une réflexion sur la vulnérabilité.

LES SENS ET LES SONS

À Pampelune, dans les Encuentros organisés en 1972 par un capitaliste espagnol basque, il y a eu un événement culturel super important sous le franquisme. On s'est demandé pendant longtemps si on collaborerait ou pas, comme beaucoup d'autres artistes. Si on ne collaborait pas, ça allait être suspendu. Il y avait des artistes internationaux comme John Cage, et on a décidé que c'était important pour l'Espagne, qu'au moins, culturellement, on puisse voir... Donc, on a décidé de participer, mais le jour où on devait faire notre action, l'ETA a mis une bombe dans une voiture tout près du théâtre où on devait faire notre concert. On s'est demandé quoi faire. Ça ne passait pas à la télévision, bien sûr. On a décidé de faire notre action. À l'intérieur, il y avait les CRS. On a commencé, sans problème; les gens étaient très excités, la liberté, l'anarchie, tout ça. Les réactions étaient souvent comme ça. Et la dernière pièce, c'était une pièce de Walter Marchetti. En France à l'époque il y avait une radio créée par des républicains espagnols exilés qui parlait des événements qui se passaient en Espagne, ce qu'en Espagne on ne pouvait pas savoir. [Car], à chaque fois qu'ils diffusaient une émission, la radio nationale d'Espagne émettait un son à la même fréquence, super fort, pour brouiller leurs informations donc on n'entendait rien. Et Walter, il avait enregistré ce son, et il l'avait monté sur six pistes, ou je ne sais pas combien de pistes, et l'action consistait... L'action se développait comme cela : on mettait sur une table au milieu de la scène une chandelle, comme celles qu'on emploie en Espagne pour la Fête des morts. On éteignait toutes les lumières du théâtre, sauf celles des sorties de secours, et on mettait ce son à une puissance inouï. Donc, ce jour-là, on a décidé de le faire, évidemment. Au bout d'un moment les flics ont obligé le directeur du théâtre à fermer les rideaux de fer qui sépare la scène du public et ils ont délogé le théâtre. Les amis étaient très excités à cause de l'attentat, nous, on était très angoissés, nous ne pouvions rien voir. Une angoisse! Mon Dieu, je me disais, s'ils commencent à être violents et s'il y a des blessés...

CB : Parce que vous étiez enfermés. 
EF : De l'autre côté du rideau de fer ! Donc, à la fin, le théâtre a été vidé calmement, rien ne s'est passé d'autre. Tu vois, ce sont des situations comme ça. Ce festival ne s'est jamais répétét ${ }^{2}$.

Esther Ferrer (née en 1937 à San Sebastián, en Espagne, vivant et travaillant à Paris) raconte ici une action réalisée par le groupe espagnol ZAJ créé en 1967 par Ramón Barce, Juan Hidalgo et Walter Marchetti. Esther Ferrer est autodidacte, les autres membres sont musiciens, et, dans le cadre du groupe, ils réalisent dès la fin des années 1960 les Conciertos $Z A J$. Les performances sont appelées « conciertos » (concerts) afin de ne pas tomber sous le coup de la censure :

Concierto $Z A J$ parce que c'est le seul spectacle qui n'était pas censuré. Si tu appelais ça Concierto $Z A J$, ils pensaient que tu jouais du piano ou du violon, ou de la contrebasse. Normalement, tous les spectacles tombaient sous le coup de la censure, la veille ou une semaine avant. Nous, on appelait ça « concert », comme ça on pouvait faire ce qu'on voulait tranquillement ${ }^{13}$.

Les Conciertos $Z A J$ comptent parmi les premières actions réalisées en Espagne, sous la dictature franquiste, auxquelles le cadre du travail collectif offre un soutien nécessaire :

C'est plus facile de faire des choses si on est trois, quatre, cinq. Faire des actions, en Espagne, cela supposait d'être dans la marge, en dehors du monde de l'art reconnu comme tel. Moi, je suis la première femme à avoir fait des performances en Espagne. C'était difficile, et c'était plus facile si on était à plusieurs ${ }^{14}$.

Dans le premier extrait d'entrevue rapporté plus haut, Esther Ferrer décrit le Concierto $Z A J$ réalisé à l'occasion des rencontres de Pampelune en 1972, un festival artistique dédié aux courants de l'art conceptuel, du happening et de la musique électroacoustique. L'événement est entre autres l'occasion de rendre un hommage à John Cage l'année de ses 60 ans. C'est la première rencontre d'Esther Ferrer avec John Cage, par lequel elle fut influencée par la suite et dont elle fut artistiquement proche, ainsi qu'avec le groupe Fluxus (dont elle définit l'approche comme «un minimalisme fondé [sur] la rigueur de l'absurde $\left.{ }^{15} \gg\right)$. Le festival, qui se déroule dans l'espace public, suscite de fortes réactions d'hostilité de la part de l'Église et des partis

\footnotetext{
${ }^{12}$ Esther Ferrer, entretien personnel, 28 mai 2015.

${ }^{13}$ Ibid.

${ }^{14}$ Ibid.

is Ibid.
} 
politiques, de droite comme de gauche ${ }^{16}$. Esther Ferrer le rappelle, l'événement est financé par une famille d'industriels proches du régime franquiste, c'est la raison pour laquelle ZAJ hésite à y participer. La pression politique qui pèse sur la performance réalisée à l'occasion des rencontres de Pampelune est lisible dans les faits décrits : attentat à la bombe, intrusion des forces de police dans le théâtre et dans l'œuvre, dénonciation de la censure dans la pièce de Walter Marchetti, obligation de fermer les rideaux de fer et isolement des artistes vis-à-vis du public. Outre les faits décrits, on peut lire aussi dans ce récit «l'excitation » du public et «l'angoisse » des artistes, qui indiquent une atmosphère émotionnellement chargée. Ce dernier mot est répété plusieurs fois avec une intonation aiguë (formalisée par le point d'exclamation), plus marquée et moins neutre que celle de la description qui précède, et appuyée par l'interjection « Mon Dieu ». L'association entre la répétition et cette sonorité particulière («Une angoisse! ») rend sensibles l'inquiétude et la frayeur ressenties par Esther Ferrer lors de cet événement. Le rythme de l'énonciation est ici très important, composé des silences (formalisés par les points de suspension) et des modulations. Il n'est d'ailleurs pas anodin que le mot «son » apparaisse deux fois, l'une des deux fois assorti d'une «puissance inouïe », et que l'œuvre décrite soit essentiellement composée d'une bande sonore diffusée à plein volume. Dans cette pièce de Walter Marchetti, le son représente à la fois un organe de propagande de la dictature (la radio nationale) et la voix des mouvements de résistance (la radio indépendante). Cette tension politico-sonore est très sensiblement transmise par Esther Ferrer à travers les modulations vocales et lexicales qu'elle donne à son propre récit. Ce récit ressenti est complété par des gestes : elle mime en effet la séparation entre artistes et public (« de l'autre côté », « de ce côté »), ce qui rend non seulement visibles mais également audibles l'isolement et la détresse temporaire des membres de ZAJ. La résolution de cette tension intervient rapidement à la fin du témoignage: le théâtre se vide «calmement », il ne se passe « rien d'autre ». Enfin, à travers un changement d'échelle temporelle important, Esther Ferrer clôt ce récit d'action : « Ce festival ne s'est jamais répété ».

${ }^{16}$ Pour une analyse détaillée de cet événement, voir Igor Contreras Zubillaga, «L'ambiguïté politique de l'avant-garde artistique sous le franquisme. L'exemple du festival Encuentros de Pampelune (1972) », dans Malika Combes, Igor Contreras Zubillaga et Perin Emel Yavuz (dir.), À l'avant-garde! Art et politique dans les années 1960 et 197o, Bruxelles, Peter Lang, coll. « Comparatisme et société », 2013, p. I09-I24. 
Le récit oral suppose une transmission des émotions par le corps, par le regard et par le grain de la voix qui est tout à fait spécifique. Les rires, les gestes, les silences, les haussements de voix, les changements de ton de même que les interjections sont autant d'indicateurs prépondérants dans l'échange direct. Ce matériau entremêlé de propriétés sonores, visuelles, émotionnelles et narratives détermine l'expérience que l'on retient des œuvres décrites. Un autre exemple peut être tiré du même entretien, à propos d'une autre action décrite par Esther Ferrer. Elle répond alors à une question sur les liens entre son travail artistique et ses engagements politiques :

EF : C'est comme le féminisme. Moi, je suis féministe 24 h sur 24 et je le serai tant que cela sera nécessaire : quand je fais de l'art, mais aussi quand je ne fais pas de l'art. En politique, c'est pareil. Je peux aller dans une manifestation, ou signer une pétition... Et dans mon travail, ça dépend. S’il arrive quelque chose qui me motive, alors je fais une performance que l'on peut qualifier de politique.

Un exemple : je devais aller au musée Reina Sofia il y a quelques années pour faire une performance, j'avais prévu de faire une performance précise et demandé du matériel au musée. Une semaine avant, je lis dans les journaux une nouvelle qui m'a absolument bouleversée : maintenant c'est beaucoup plus, mais à l'époque c'était I86I ou I863 migrants, qui étaient morts d'une mort violente en essayant de rentrer par les frontières de l'Union Européenne ${ }^{17}$. Moi, ça me rend malade, parce que je suis aussi une migrante. Alors, je me dis, il faut faire quelque chose. Je me dis, ces migrants, personne ne les connaît, pour nous ce sont des anonymes, il faut que je fasse en sorte que les gens se rendent compte de cette tragédie. Chose qui me semblait valable. J'ai téléphoné [au commissaire de l'exposition] et j'ai dit: «Je ne vais pas faire la performance prévue, je vais faire autre chose. » J'ai demandé des volontaires, qui se sont présentés au musée, 23 personnes se sont portées volontaires. J'ai dit au public: « On va simplement compter un par un les numéros des morts, si vous voulez vous pouvez le faire avec nous. » J'ai expliqué les circonstances de leurs morts. « Pour vous, c'est un chiffre, I86I, mais on va compter un à un. Pour se rendre compte vraiment de la tragédie que ça suppose. » Et on a fait ça. Ça a duré longtemps.

${ }^{17}$ Esther Ferrer fait référence au nombre de migrants morts en 1972 sur les rives de la Méditerranée. À titre indicatif, ce nombre s'élève pour 2016 à plus de 3000, selon l'OIM : «Mediterranean Migrant Arrivals in 2016 : 227,316; Deaths : 2,920 », mis en ligne le 7 mai 2016, https://www.iom.int/fr/news/mediterranean-migrant-arrivals-2016-227316-deaths-2920 (consultation le 2 juin 2017). 


\section{$\mathrm{CB}:$ Combien de temps?}

$\mathrm{EF}$ : Presque une heure, même plus. J'ai demandé de compter comme une litanie: «Un... deux... trois... quatre... » Jusqu'à la fin. J'ai dit, normalement, pour les victimes du terrorisme, on garde une minute de silence. Pour moi, c'est vraiment un terrorisme capitaliste qui a fait ces morts, c'est encore plus horrible. Donc, j’ai dit : « On va se lever, garder une minute de silence. »

Voilà. Mais ça, je le fais quand j’ai une idée. Et je ne pense même pas à faire un acte politique. Ce sont mes tripes ${ }^{18}$.

On trouve des descriptions de cette même performance dans des entretiens publiés, par exemple ici dans un entretien d'Esther Ferrer avec Medhi Brit et Sandrine Meats, publié en octobre 2014 :

Si l'origine de la performance est politique, quand il s'agit de l'immigration, de la position des femmes, je veux absolument que les gens comprennent. Si je proteste contre quelque chose, je veux que ça soit clair. C’est le cas, par exemple, de la performance que j’ai faite lorsque j'ai été invitée au Reina Sofia de Madrid. Quelques jours avant, j’avais lu dans la presse un article relatant la mort violente, l’année précédente, de 1863 immigrés clandestins décédés en essayant de traverser une frontière européenne. J'ai expliqué au public les raisons de cette performance et leur ai dit que nous allions compter un à un pour nous rendre compte de la tragédie que cela représentait. J'ai commencé avec des volontaires trouvés au préalable, et le public nous a rejoints. Nous avons compté jusqu'à 1863 , cela a duré une heure. C'était une sorte de psalmodie pour les morts, qui pour nous n'avaient ni noms ni existence réelle; ils étaient seulement un nombre comme s'ils n'avaient ni famille ni relations. Je voulais que, pour un moment, ils existent. Je voulais nous faire prendre conscience que c'est « notre » Europe qui permet çâ' ${ }^{19}$.

Plusieurs choses sont frappantes dans la comparaison entre les deux récits : d'abord, leur ressemblance dans le contenu et dans la formulation. Pour commencer, l'exemple de cette performance intervient dans la même ligne d'argumentation, c'est-à-dire quand il s'agit pour Esther Ferrer d'expliquer son rapport avec l'engagement politique (dans les deux cas, les sujets de l'immigration, de la position des femmes ou du féminisme sont les causes pour lesquelles elle peut envisager de faire une «performance politique »). L’entretien n'échappe donc pas à une forme

\footnotetext{
${ }^{18}$ Esther Ferrer, entretien personnel, 28 mai 2or5.

19 Brit et Meats, 2014, p. 184 .
} 
de standardisation des énoncés. On trouve aussi des récurrences dans les termes employés : la performance vise à se « rendre compte »d'une «tragédie », et, pour cela, le nombre de migrants décédés a été égrené « un à un ». La récurrence de la locution adverbiale est importante, car elle est la modalité à travers laquelle le cour de la performance est introduit et elle indique un rythme dans la succession des chiffres énoncés collectivement. Ce rythme, suggéré par la forme écrite, est tout particulièrement sensible à travers le récit oral: d'abord, la locution est répétée (« un par un », puis «un à un »), et la prononciation en est particulièrement soignée (Esther Ferrer ponctue en effet ses deux phrases en s'arrêtant un temps sur cette locution, qui constitue en quelque sorte un pivot sonore). Cela s'intensifie et se cristallise au moment du décompte dont elle restitue dans son récit les quatre premiers temps («Un... deux... trois... quatre... »), et ce, à une vitesse qui imite celle $\mathrm{du}$ direct, c'est-à-dire en veillant à ce que le rythme de l'énonciation pendant l'entretien soit le même que celui qui a été adopté pendant la performance. C'est à travers ce rythme qu'elle transmet, avec l'ambiance sonore de l'action, un aperçu de son ressenti et de celui des participants. Encore une fois, le champ lexical du son est présent dans le récit, à travers la « minute de silence ». Les modulations, le chant (tantôt évoqué par le terme de « litanie, tantôt par celui de « psalmodie ») à travers lesquels elle module sa voix permettent de réactiver une atmosphère sensible, dans un échange qui ne peut advenir qu'à travers la coprésence physique qui est propre à l'entretien.

On voit donc que le récit fait pendant l'entretien, s'il ne peut pas être considéré comme un reenactment dans la mesure où il ne s'agit pas de refaire l'œuvre mais de la raconter, comprend des éléments de reprise très manifestes, qui permettent de différencier le témoignage vivant du document. D'une part - et c'est particulièrement approprié dans le cas de l'œuvre d'Esther Ferrer - le récit restitue une facette précieuse de l'ambiance sonore des actions : durée, rythme et ton sont au cœur de la matière orale. D'autre part, il transmet les sensations et les émotions relatives à l'expérience subjective de l'artiste, du narrateur ou de la narratrice: l'excitation, l'angoisse, la tension sont réactivées par le récit autrement qu'elles ne le sont par le support documentaire. La narration vivante sollicite non seulement le regard de la personne à qui elle est adressée, mais aussi son ouïe et plus généralement sa physicalité. 


\section{REGARDER EN SILENCE}

Durant notre échange, Esther Ferrer ne fera quasiment pas appel au matériau visuel lié aux performances. Bien que le récit et le ressenti fassent efficacement circuler les sensations, l'absence d'images contribue au caractère flou des contours des ouvres; il me faut donc composer mentalement pendant l'entretien des représentations visuelles «à l'aveugle» (espace, participants, mouvements). Le phénomène contraire peut se produire quand l'artiste mobilise de la documentation pendant l'entretien. C'est le cas dans le commentaire qui va suivre, recueilli lors d'un entretien avec Nil Yalter (née en 1938 au Caire, en Égypte, vivant et travaillant à Paris) qui raconte les activations récentes d'Exile is a Hard Job (C'est un dur métier que l'exil), une pièce qu'elle a présentée pour la première fois à l'A.R.C. au Musée d'art moderne de la Ville de Paris en 1983. L'A.R.C. (Animation Recherche Confrontation) est un lieu d'exposition qui vise la promotion de courants expérimentaux en art contemporain comme l'art conceptuel, le minimalisme et l'art vidéo. Nil Yalter fait alors partie des pionnières en matière d'art vidéo en France, et Exile is a Hard Job est présenté en 1983 sous la forme d'une installation de moniteurs vidéo assortie de textes, de photographies et de peintures. La phrase « C'est un dur métier que l'exil » est inscrite sur les murs de l'A.R.C. (voir la figure I), côtoyant une vidéo d'entrevues avec des travailleurs turcs dans des magasins de prêt-à-porter de la rue du Faubourg-Saint-Denis à Paris ${ }^{20}$.

Depuis 1983, cette œuvre a fait l'objet de plusieurs réexpositions et réactivations, ce que Nil Yalter appelle, nous le verrons, «faire du nouveau avec d'anciens travaux ». Exile is a Hard Job - les vidéos, les photographies, les textes et les peintures initialement exposés à l'ARC - a par exemple été réexposée au Musée de l'histoire de l'immigration à Paris en $2009^{21}$. Le commentaire ci-dessous renvoie à des reprises encore plus contemporaines ayant pris une autre forme, plus proche de

${ }^{20}$ Fabienne Dumont, « Feminists, Immigrants, Workers: Struggle's Connection », dans Derya Yücel, Juan Vicente Aliaga, Fabienne Dumont et Melis Tezkan, Nil Yalter, Istanbul, Galerist /Berlin, Revolver Publishing, 2013, p. 2II.

${ }^{21}$ Exile is a Hard Job III, Musée National de l'histoire de l'immigration, Paris, 2009. Exposition documentée sur le site internet de Nil Yalter: http://www.nilyalter.com/works/sI / exile-is-a-hard-job-iii-2009.html (consultation le 7 septembre 20I7). 


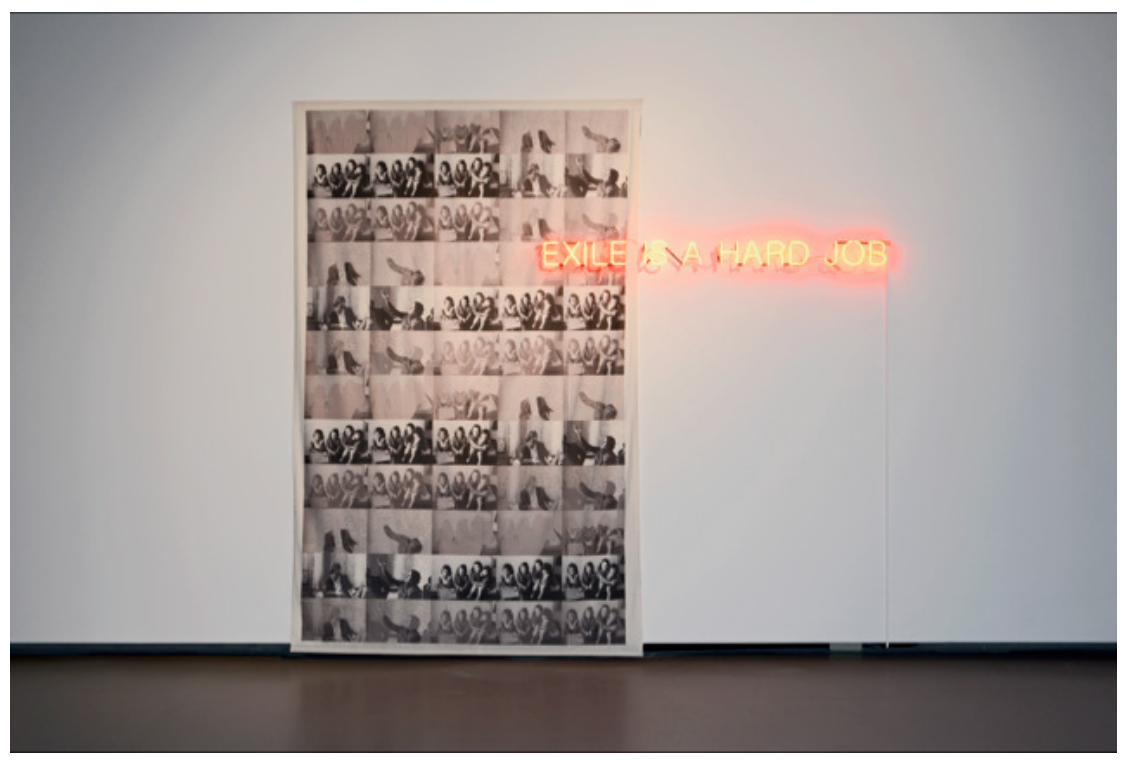

Fig. I. Nil Yalter, Exile is a hard job, Drap, Fondation Hermès Bruxelles, 1977 / 2015. Avec l'aimable autorisation de l'artiste. (C) Isabelle Arthuis

l'action, car elle comprend un temps d'affichage dans les villes où l'artiste expose. Elle décrit ainsi ces reprises :

NY: J'ai pris le rythme de faire des nouveaux travaux avec mes anciens travaux. [Rires] Je fais des affichages sauvages dans la rue.

$\mathrm{CB}$ : En ce moment?

NY: Oui. Dans le monde entier. Je ne suis même pas obligée d'y aller, j'envoie une image très haute définition, et ils font des affiches eux-mêmes.

$\mathrm{CB}:$ La galerie ?

NY : Oui, la galerie, ou les gens, les jeunes gens. Je confie ça à tout le monde. À partir d'une seule image, avec la même phrase dessus « C'est un dur travail que l'exil », et c'est tout, dans le monde entier. Et pour moi c'est un nouveau travail. [...]

$\mathrm{CB}:$ Est-ce qu'il y a des traces?

[N. Y. cherche les photographies dans son ordinateur et me les montre.]

NY : Ça, c'est la première. Toujours dans la langue de l'endroit où je le fais. Voilà, ils ont fait les affiches. Chaque pays réagit différemment. Voilà... C'est moi-même qui affiche ça aussi la nuit. Un soir, après avoir affiché, on est partis pour dîner et, quand on est revenus, les gens du quartier, qui commence à être un peu huppé, avaient déchiré toutes les affiches. On a tout réaffiché pour le lendemain, à ce moment-là, c'est la police qui [...] a tout déchiré.

$\mathrm{CB}$ : Pouvez-vous m'en dire plus sur ces photographies, sur ces images ? 
NY : C'est toujours la même image, je l'envoie partout. Regardez bien. [Elle cherche dans ses fichiers numériques.]

Voilà. On l'a fait à Vienne, avec la galerie. Ça, c'est la galerie. On a commencé en en mettant à l'intérieur. Il y a un gars qui est venu - c'est un quartier qui était populaire, qui est devenu très chic parce qu'il y a tous les musées autour - , qui a hurlé à mon galeriste en disant : «On ne veut pas des Turcs, des immigrés dans notre quartier. Enlevez ça. » Il a hurlé, hurlé, hurlé, il est parti. Il [le galeriste] a été obligé de les enlever. Après, on est allés dans le quartier turc et pakistanais. Alors, là, on a facilement mis toutes les affiches.

[Elle montre.]

Voilà... C'était plus petit, ces affiches. Après, il y a eu des réactions, ils ont déchiré aussi un petit peu... Donc, ça c'en est une, mais la meilleure, mais la meilleure pour moi, c'est à Bombay. Je n'y suis même pas allée. Voilà Bombay. Ça, c'est Clark House, un musée. Ils ont mis des affiches dans le musée, en hindou et en anglais. Et puis dans les rues. Mais comme il n'y a pas beaucoup de murs, ils en ont mis dans les bicoques comme ça. [Nous regardons en silence.]

NY: Sur les autobus qui se baladent... toujours la même affiche. Pour l'instant, il n'y a que trois villes, mais maintenant je continue ${ }^{22}$.

Dans cette description, Nil Yalter mentionne les activations de C'est un dur métier que l'exil qui ont eu lieu à Valence (Espagne), à Vienne et à Bombay ${ }^{23}$. En disant faire « du nouveau avec des anciens travaux », elle inscrit bien ces actions dans le prolongement des versions précédentes de la pièce portant le même titre, jusqu'à celle de 1983. La pièce a aujourd'hui un aspect performé beaucoup plus important que dans l'installation initiale: outre l'envoi et l'impression des affiches, la majeure partie de l'œuvre consiste à aller les coller dans l'espace public et à y inscrire dessus à la peinture rouge cette même phrase dans la langue du pays qui l'accueille ${ }^{24}$ (voir la figure 2). Il arrive que Nil Yalter participe aux réactivations comme ce fut le cas par exemple à Valence («C'est moi-même qui affiche ça aussi la nuit »), mais celles-là peuvent aussi avoir lieu en son absence («Je ne suis même pas obligée d'y aller »), comme à Bombay.

${ }^{22}$ Nil Yalter, entretien personnel, 22 juillet 20I5.

${ }^{23}$ Depuis l'entretien, la dernière réactivation en date a eu lieu à Metz à l'occasion de la rétrospective de l'artiste au 49 Nord 6 Est (Fonds régional d'art contemporain de Lorraine), du 5 février au 5 juin 2016. Tiphanie Chauvin, Béatrice Josse, Alexia Tirelli, Nil Yalter, Metz, 49 Nord 6 Est-Fonds régional d'art contemporain de Lorraine, février-juin 2016.

24 « Exile is a Hard Job » est le titre d'un poème du poète turc Nâzim Hikmet, C'est un dur métier que l'exil: anthologie poétique, traduction de Münevver Andaç, Paris, Le temps des cerises, 1999. 


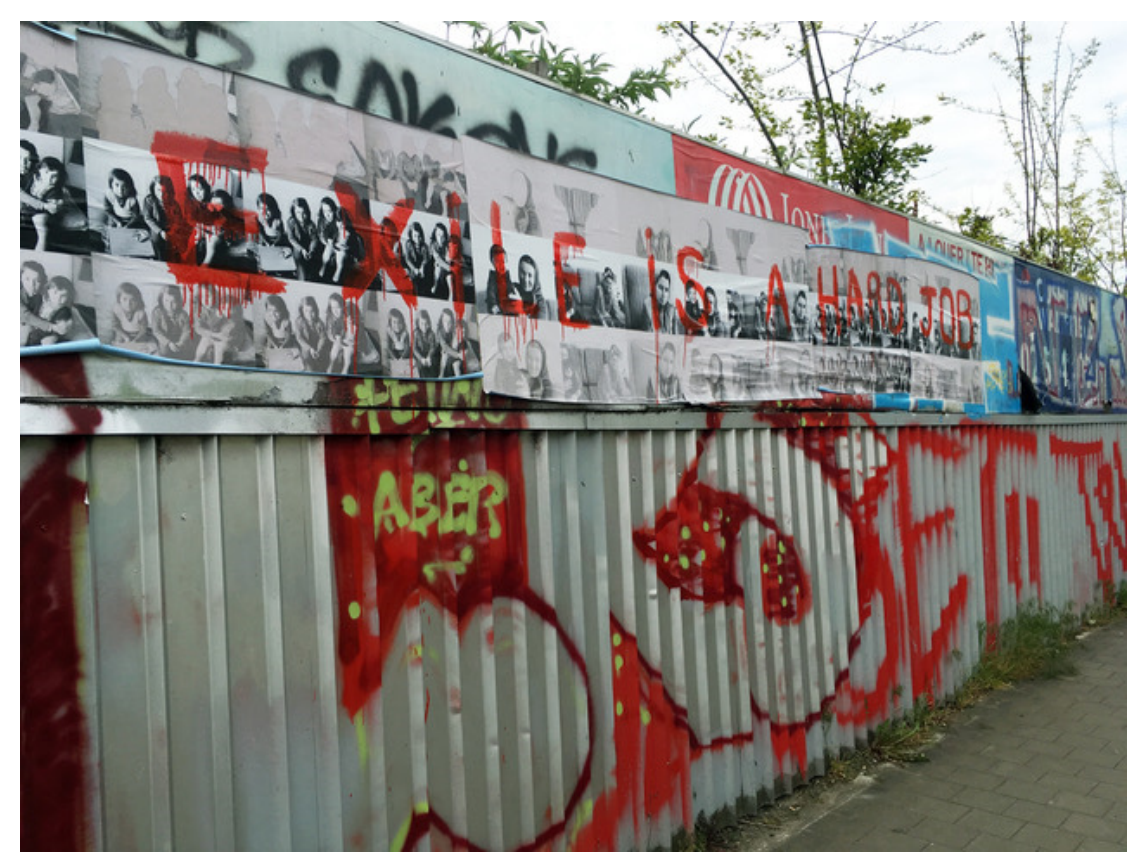

Fig. 2. Nil Yalter, Exile is a hard job, Affichage, WIELS Bruxelles, 1977/2017. Avec l'aimable autorisation de l'artiste. @ Zoé Schreiber

C'est en réponse à une question de ma part (« Est-ce qu'il y a des traces ? ») que Nil Yalter va chercher des photographies, dont la présence pendant notre échange devient vite centrale. Autour de la documentation s'articule une chorégraphie de gestes qui contribuent tantôt à établir un lien entre les images et le récit, tantôt à se substituer à ce dernier. Dans la retranscription, ces gestes sont indiqués sous forme de didascalies, entre crochets: Nil Yalter «cherche » les images, puis elle les «montre», et nous les «regardons». D'autres gestes interviennent dans le récit qui ne sont pas indiqués dans la retranscription, ce sont ceux qui viennent avec les expressions « ça », « comme ça », « voilà » : quand elle les prononce, elle pointe les images du doigt. Ce sont des expressions vides, qui agrémentent les gestes et dirigent les regards. Grâce à elles, l'artiste dirige en effet mon regard vers les représentations visuelles à partir desquelles elle suggère que je me fasse ma propre idée. Ces gestes-qui-montrent oscillent entre une fonction de complément du récit et une fonction de substitut. Ils sont complémentaires quand le récit donne une information sur l'image commentée («ça, c'est la première »), mais ils le supplantent quand les informations ne sont pas données. Cela est très 
évident quand, plutôt que de raconter l'origine ou l'histoire de l'image (« Pouvezvous m'en dire plus sur ces photographies, sur ces images ?»), Nil Yalter dit: «Regardez bien », comme si les informations se trouvaient dans la représentation visuelle, ou comme si les informations visuelles étaient plus porteuses de sens que les informations orales. Point culminant de ce dispositif de description, il arrive même que nous les regardions «en silence ». On voit apparaître ici une modalité de transmission très différente de celle qui opère à travers la seule matière sonore : le registre visuel est nécessaire pour la description proposée ici par Nil Yalter. À la différence d'Esther Ferrer, qui transmet les sensations de l'action en transformant en paroles ses souvenirs, dans le cas de ce récit précis, Nil Yalter les transmet en en montrant des traces matérielles. C'est à travers elles qu'elle revoit et revit l'action. Il me semble alors que je l'accompagne en train de se remémorer et que je participe à cette remémoration par la coprésence et par le regard, plutôt que par l'appropriation mentale d'une narration. La coprésence et le fait de passer un moment ensemble sont essentiels pour caractériser l'entretien et ses vertus méthodologiques, car ils permettent aux deux interlocuteurs de faire des choses dans un même temps, en même temps: regarder, entendre, sentir. Cet échange immatériel implique des formes d'engagement spécifiques, de la part de la narratrice et de la part de la personne qui écoute.

Il me paraît pertinent ici de mobiliser une terminologie liée à la vulnérabilité et à la précarité telle qu'elle est théorisée, entre autres, par Judith Butler. Je crois en effet qu'on peut poser à profit, avec la philosophe, la question des formes de subjectivité et de vie « envisagées ${ }^{25}$ » dans ce genre d'entretien. La vie précaire telle qu'elle la conceptualise implique un sujet toujours déjà pris dans un rapport essentiel à l'altérité, et dont la dépendance à l'autre met en crise sa propriété de souveraineté. La précarité a donc la capacité de défier les formes dominantes de représentation. Pour Judith Butler, il y a une forme de responsabilité éthique spécifique à «faire face » à l'autre, c'est-à-dire à accueillir la représentation de son existence en même temps que la disjonction qui rend cette représentation impossible. Le caractère manquant et partiel des images et des ressentis qui accompagnent les récits au sujet des performances est l'une de leurs propriétés

${ }^{25}$ Judith Butler, « Precarious Life », dans Judith Butler, Precarious Life, Londres, Verso, 2004, p. I28-I5I. Le face-à-face avec l'autre est essentiel dans la conception butlérienne de la précarité et des vies précaires, qu'elle développe en particulier à partir des écrits d'Emmanuel Levinas sur le visage (face). 
centrales: pour survivre, les œuvres sont essentiellement dépendantes de la circulation des récits et des images qui les tracent, et pourtant celles-là échouent fondamentalement à les transmettre complètement. Cette dépendance est tout particulièrement révélée par l'entretien, car l'incomplétude et l'irrégularité des témoignages y sont centrales. Cette forme de recueil implique aussi une responsabilité spécifique de la part de la personne qui écoute : sa posture doit être empreinte d'une concentration, d'une attention et d'une empathie qui ne sont pas pareillement requises face à des matériaux inanimés. Enfin, la vulnérabilité qui relie les acteurs de la relation permet aussi de penser le sujet de la discussion, c'est-à-dire l'œuvre. En effet, une performance reprise dans un récit est une œuvre ouverte, perméable, incarnée dans une parole, qui conçoit une réception subjective, partielle et tout aussi incarnée dans une écoute et des questions. L'échange oral présente donc cette forme de perméabilité et d'affectivité qui ressemble, sous certains aspects, à la performance.

\section{Autour des GuVRes: UNe histoire des OUBLIS ET DES INCERTITUDES}

Les incertitudes sont une composante essentielle des œuvres de performance telles qu'elles sont reprises et présentées dans l'entretien : les artistes émettent des doutes quant aux contours spatiaux et temporels des œuvres, montrent une fragilité quant aux conséquences matérielles des réactions et une incertitude quant à la capacité du travail artistique de procurer une sécurité économique. Tous ces éléments contingents et marginaux prennent une place centrale dans le récit oral et permettent d'envisager l'incertitude à double-titre : comme un principe temporel et comme un principe économique. Ils invitent à penser la performance comme véhicule d'une temporalité non linéaire et d'une matérialité précaire, ce qui caractérise les reprises (replay) telles que les analyse Rebecca Schneider :

The explicit replay of a time-based art troubles the prerogatives of singular artists. The assumptions of forward-marching time, and the frontiers-driven narratives of capital that-like a great perspective machine-invests in the linear geometry of vanishing points. Touching time against itself, by bringing time again and again out of joint into theatrical, even anamorphic, 
relief presents the real, the actual, the raw and the true as, precisely, the zigzagging, diagonal, and crookedly imprecise returns of time ${ }^{26}$.

Pour Rebecca Schneider, la temporalité propre aux reprises de performance a plusieurs propriétés : d'abord, elle s'oppose à une perspective linéaire et téléologique (la reprise «trouble » la « «marche en avant » du temps); ensuite, sur le registre spectaculaire de la «théâtralité », elle vide l'activité artistique de son ontologie (la reprise donne à voir le « réel », l'actuel, le vif ou le vrai comme des « retours », c'est-à-dire comme ayant toujours déjà un statut de copie). Ces critères théoriques s'allient à des qualités formelles, comme un « relief » « anamorphique », c'est-àdire renversé, et un aspect «zigzagant», « diagonal». Rebecca Schneider caractérise également cette temporalité à l'aide d'un vocabulaire corporel, évoquant à cet effet un temps « nerveux » et « syncopé 27 ». Cette réflexion s'articule dans un paysage épistémologique construit par les pensées féministes en histoire de l'art et, plus récemment, par les perspectives queer, dont j'avance ici qu'elles sont tout particulièrement cohérentes avec la performance.

DOUTES, ERREURS, OUBLIS

Dans le récit de Nil Yalter, aucune date n'est associée aux différentes reprises d'Exile is a Hard Job; seules les villes sont précisées. Les photographies indiquent des reprises plutôt récentes, mais la chronologie des actions présentées n'est pas spécifiée par l'artiste. D’une manière générale, il arrive très souvent dans un entretien qu'il y ait un certain flou au sujet des dates, et ce flou peut être envisagé comme un levier pour analyser ses enjeux méthodologiques. En effet, outre la place accordée aux émotions, une autre caractéristique du récit oral est la place centrale qu'il permet de donner aux doutes et aux hésitations.

Dans le témoignage d'Esther Ferrer, on peut relever plusieurs approximations au sujet de la performance au Reina Sofia : elle est réalisée « il y a quelques années » au moment de l'entretien, le nombre exact de morts dont le décompte a été fait varie (I86I, I863 dans le premier; I863 dans le second). En dehors de la description de ces deux seules ouvres, il arrive de nombreuses fois au cours de l'entretien que l'artiste hésite, qu'elle se reprenne au sujet de la date ou du lieu d'une performance ou d'une exposition. Elle hésite par exemple sur l'âge qu'elle avait lors

\footnotetext{
${ }^{26}$ Rebecca Schneider, Performing Remains. Art and War in Times of Theatrical Reenactment, Abingdon, Routledge, 20II, p. I6.

27 Ibid., p. 89.
} 
de sa récente rétrospective au MAC $\mathrm{VAL}^{28}$. Il arrive souvent qu'elle dise « je ne me souviens plus », «je ne me rappelle plus», c'est-à-dire qu'elle formule explicitement l'oubli. Elle est d'ailleurs très lucide au sujet de ces approximations :

Le temps, ça a toujours été quelque chose que j'ai travaillé, et pourtant je ne sais jamais l'heure, le jour, etc. Pour remplir un chèque, je dois demander en quelle année on est, parfois je ne sais pas si c'est I4 ou is.

Cette place laissée à l'oubli et aux doutes quant aux dates dans son énonciation est valable aussi pour les œuvres, à propos desquelles l'approximation devient même un geste explicite :

Comme je ne mets pas de date, je ne sais jamais quand j'ai fait les choses. Même dans mon travail plasticien, je ne date pas les choses. Je ne signe pas, je ne mets pas de dates. Maintenant, dans les expositions, ils t'obligent à mettre des dates, alors quand j'expose, je mets des dates approximatives. Pour les performances, je sais quand je les ai faites parce que — pas toujours, mais souvent - je l'écris dans mon CV, alors je sais quand je l'ai faite. Je ne sais jamais quand je les ai écrites ${ }^{29}$.

Esther Ferrer revendique une conception de la performance qui ne soit pas définie par des dates ou des lieux spécifiques. Elle ne fait donc pas d'effort pour se les remémorer ni pour les transmettre — pas plus dans le cadre d'un entretien que dans celui d'une exposition. Les coordonnées spatiales et temporelles de ses performances ne sont pas des critères importants à ses yeux, et peuvent donc varier. Le doute et la réécriture, au cœur de la définition de la performance pour Esther Ferrer, sont très présents dans son témoignage. Les contours précis des œuvres sont volontairement floutés, tandis que l'idée qui est au cœur de la performance est transmise avec précision et rigueur.

La place de l'oubli et de l'erreur est un enjeu important de la reprise opérée en entretien : la remémoration orale et en temps réel implique en effet des errances mémorielles qui doivent être intégrées comme des composantes temporelles à part entière de l'œuvre transmise. À travers ses hésitations et ses imprécisions répétées, Esther Ferrer performe une temporalité qui n'est ni linéaire ni assurée : l'entretien nous plonge bien dans ce rythme mémoriel « zigzagant, diagonal, et facétieusement

\footnotetext{
${ }^{28}$ « Face b. Image/Autoportrait », au MAC VAL, Musée d'art contemporain du Val-deMarne, Vitry-sur-Seine, is février-13 juillet 2014.

29 Esther Ferrer, entretien personnel, 28 mai 20I5.
} 
imprécis $^{30} \gg$, que Rebecca Schneider attribue au reenactment. Se ralliant ainsi aux qualités formelles de la reprise telles que les caractérise Rebecca Schneider, le récit oral permet d'accueillir ces facéties du temps.

ANECDOTES, CIRCONSTANCES, CONTINGENCES

Le dispositif de l'entretien convient particulièrement bien à la transmission d'une conception des œuvres dans laquelle un certain type d'informations s'efface, tandis que d'autres s'avancent et deviennent plus prégnants. Les informations factuelles comme les dates ou les lieux, autrement dit ce qu'un document a vocation à préciser la plupart du temps, s'effacent. Ces oublis laissent la place à la remémoration d'un autre type d'informations, et en particulier à l'évocation des circonstances de la réalisation, c'est-à-dire de la production et de la réception des œuvres.

Cela est tout à fait présent dans le témoignage de Nil Yalter. Son récit des reprises d'Exile is a Hard Job, dont j'ai relevé qu'il était très peu empreint de contenu au sujet de la forme même de l'action, est en revanche empli d'anecdotes sur les conditions de la réception des affichages sauvages, notamment les dégradations dont ils ont fait l'objet. Les endroits du récit où sa narration est la plus développée et détaillée sont ceux qui concernent les « réactions », différentes dans chaque contexte («Chaque pays réagit différemment »), mais la plupart du temps agressives. Les affiches qui sont au cœur de la performance ont maintes fois été abîmées par des usagers de l'espace public dans lequel elles ont été disposées. Lors de la reprise à Valence, ce sont « les gens du quartier », puis la « police », qui les ont déchirées. À Vienne, un habitant du quartier venu «hurler» des paroles xénophobes est parvenu à ce que la galerie Hubert Winter (qui représente Nil Yalter à Vienne) retire les affiches de leur lieu d'accrochage; dans le quartier turc et pakistanais de cette ville aussi, les affiches ont été déchirées, mais dans une moindre mesure (《un petit peu »).

Ces détériorations font partie de l'œuvre telle qu'elle est présentée dans son témoignage et tiennent même une place plus importante que ne tient la description du geste précis de l'affichage. On peut rejoindre ici les réflexions développées plus tôt au sujet de l'énonciation des émotions dans le témoignage d'Esther Ferrer : chez Nil Yalter aussi, on trouve un effet de répétition («il a hurlé, hurlé, hurlé ») qui

\footnotetext{
${ }^{30}$ Schneider, 20II, p. I6.
} 
met l'accent sur la violence sonore et physique de la réaction décrite : un habitant du quartier qui fait intrusion dans la galerie et qui parvient à ce que ses œuvres en soient retirées. Alors qu'Esther Ferrer évoque sa propre « angoisse », la mention des émotions chez Nil Yalter passe peut-être ici par le récit de la réaction d'un autre. En tout cas, comme pour le Concierto $Z A J$, ce sont des éléments du contexte qui prévalent dans la description de l'œuvre. Exile is a Hard Job, telle que Nil Yalter la décrit, est formée et informée par les gestes des usagers hostiles. En d'autres termes, on peut considérer que la matière narrative de l'œuvre est ici essentiellement constituée par la mention de ses conditions de production et de réception, et plus spécifiquement de sa dégradation. Du fait de l'ouverture qu'il laisse à la mention de ces conditions, l'entretien montre donc des œuvres vulnérables, fragiles et contingentes. L'anecdote, les circonstances en marge de l'action, peuvent être situées au centre de sa narration.

\section{PRÉCARITÉS}

À travers l'oralité, il s'agit donc de déplacer au centre du récit un matériau narratif traditionnellement périphérique. Cela s'applique aux œuvres dans leur singularité comme on vient de le voir dans le récit de Nil Yalter, mais aussi à l'œuvre dans sa généralité. Pendant l'entretien se découvrent non seulement les conditions dans lesquelles les œuvres ont été produites, mais aussi celles dans lesquelles les artistes vivent et travaillent. Le récit oral invite à prendre en compte les conditions matérielles comme un composant essentiel de l'œuvre — c'est-à-dire du travail — performatif. Les deux artistes interrogées décrivent la précarité des conditions matérielles dans lesquelles elles ont exercé pendant la majeure partie de leur parcours professionnel. Esther Ferrer raconte ainsi les nombreux « petits boulots » qu'elle a exercés pour gagner de l'argent, activités tout à fait étrangères au milieu de l'art :

EF : J'ai tout fait. J'ai peint des murs, j'ai fait des chantiers avec un groupe d'anarchistes espagnols qui étaient des migrants. J'ai travaillé avec eux, on peignait les murs des maisons à l'intérieur, en bleu, en rose. Je le fais très bien. J'ai gardé des enfants, j'ai nettoyé des maisons. J'ai fait beaucoup de traduction aussi, du français vers l'espagnol.

Nil Yalter se rappelle la petitesse des espaces de travail dans lesquels elle a construit Toprak Ev, une installation qui a été exposée en 1973 au Musée d'art moderne de la Ville de Paris.

CB : Ce que vous faisiez, vous, à cette époque-là, c'était sans moyen ? 
NY : Sans moyen, sans un sou ! On n'avait pas un sou ! [...] Il n'y avait pas d'équipement, il n'avait pas d'argent, il n'y avait pas d'espace. J'ai vécu et travaillé pendant deux ans dans un espace de i 8 mètres carrés pour faire la tente de nomade qui, elle-même, est de 9 mètres carrés.

Elle indique plus loin que cette exiguïté a probablement eu une importante influence sur son choix de faire de la vidéo, un médium qui prend peu de place. Il y a une corrélation entre le choix de médiums légers et ces conditions de travail précaires. D'autres facteurs contribuent à la précarité des artistes : l'indifférence $d u$ milieu artistique parisien envers les pays dont elles ont choisi d'émigrer (Esther Ferrer arrive d'Espagne en 1973, et Nil Yalter, de Turquie la même année) :

NY : Quand on est arrivés en 1973, ils ne savaient même pas ce que c'était, la Turquie. Les artistes turcs, ils ne connaissaient même pas.

EF : En France, on n'a jamais pris trop au sérieux l'art des Espagnols. Sauf celui qui était consacré par le système, mais ça n'était pas nous.

Les deux artistes soulignent aussi que cette indifférence, qui est une forme de discrimination, est aussi liée au genre féminin :

NY: Comme beaucoup de femmes artistes, on n'avait aucun compte à rendre, de toute façon, personne ne s'intéressait à nous. Donc, on n'avait aucun tabou, on faisait ce qu'on voulait, on était très libres.

Nil Yalter fait un lien entre le désintérêt, voire le mépris du milieu de l'art pour le travail des artistes femmes et une forme de liberté, donc d'autonomie dans la création : la précarité subie est associée à une forme d'indépendance revendiquée. Il est difficile d'établir un lien de causalité dans un sens ou dans l'autre, mais le choix de la performance est souvent aussi associé à un rejet du marché de l'art, c'est-à-dire de la vente d'objets. Les deux artistes décrivent par exemple leur refus de s'associer à une galerie au début de leur carrière :

NY : En 20Io, j'ai eu une galerie. Avant, je n'avais jamais eu de galerie, mais j'étais contre les galeries. Ça aussi, ça faisait partie du milieu de l'art, la vieille galerie... Ça faisait partie de ma démarche sociologique.

La galerie représente le milieu de l'art classique que la démarche artistique de Nil Yalter vise à déconstruire et à critiquer. Le terme « sociologique » est ici employé pour désigner un positionnement en dehors ou à côté des mécanismes marchands. Esther Ferrer décrit ce même rejet qui perdure encore maintenant, même si elle est aujourd'hui représentée (par exemple par la galerie Lara Vincy à Paris). Elle parle de la vente de ses œuvres comme de quelque chose d'extrêmement difficile : 
EF : Je ne vends jamais directement. C'est la galerie. J'ai horreur de ça, je ne peux pas, ça me met mal à l'aise. Je l'ai fait une fois, et je me suis juré de ne jamais plus le faire. Même à mes bons amis, je dis d'aller à la galerie. [...] Tu vois, en réalité, ce qui m'intéresse dans la pratique artistique, c'est le fait de travailler dans mon atelier. C'est l'unique chose qui me procure du plaisir.

Légère, discrète, la performance répond donc simultanément à des conditions de travail précaires et à une opinion critique vis-à-vis de la marchandisation alliée à une forme d'autonomie dans le travail.

NY: Vous savez, à mon âge, ne pas s'inquiéter pour le peu de temps qu'il reste pour vivre, c'est quand même mieux.

$\mathrm{CB}$ : Aujourd'hui, accordez-vous de l'importance au confort matériel ?

NY: Oui, c'est quand même important. Quand c'est arrivé [la reconnaissance], je n'avais à nouveau plus un sou [Rires]. Je n'en croyais pas mes oreilles quand ça a commencé à marcher ! Donc, j'étais contente mais... toutes ces foires d'art, je trouve ça nul... À part une ou deux. Ça ne me plaît pas beaucoup. C'est pour ça que je préfere des choses comme à Bombay où il n'y a rien à vendre, rien à garder, on n'a même pas besoin d'y aller, et ça ne coûte rien. Alors ça, ça, c'est ma grande joie.

Pendant les années 1970 et 1980, la performance permet à ces artistes de produire des œuvres avec peu de moyens et dans des espaces exigus, tout en échappant aux circuits marchands. Esther Ferrer dit aussi le désintérêt qu'elle a eu pendant longtemps vis-à-vis du dispositif même de l'exposition :

$\mathrm{CB}$ : Comment fait-on pour vivre de la performance?

$\mathrm{EF}$ : Je n'en vis pas. J'ai presque 78 ans, et je vis de mon travail artistique depuis is ans. Et surtout, pas seulement de la performance... Pendant longtemps, je n'ai pas exposé parce que ça ne m'intéressait pas, et personne ne me le demandait, ce qui était très bien. Mais à partir de 1983 ou 1984, en Espagne, il y a eu une exposition qui s'appellait Fuera de formato ${ }^{31}[\ldots] \mathrm{ZAJ}$ a exposé et, à partir de ce moment-là, j'ai commencé à exposer. Ils ont commencé à me demander d'exposer et tout ça. Et j'ai exposé, mais très peu. Et puis, j'ai gagné tous ces prix en Espagne et en France, tu commences à être connue, les gens te demandent plus de choses, les galeristes ont commencé à vendre, j'ai commencé à vendre. Mais c'est surtout parce que les galeries ont pu vendre le travail de plasticien. Et j'ai pu commencer à en vivre. Encore

${ }^{31}$ « Fuera de formato », au Centro Cultural de la Villa, Madrid, février-mars 1983. 
aujourd'hui, si je devais vivre seulement de mon travail de performance, je ne pourrais pas.

L'entretien est l'occasion de revenir sur les étapes de construction de la trajectoire professionnelle et de souligner que la performance n'est pas rentable sur le plan économique - que c'est une pratique « à perte ». La matière narrative est modelée par les incertitudes quant aux réactions et aux réceptions, qu'il s'agisse de celles des spectateurs ou de celles des acteurs du marché de l'art. La précarité des œuvres et des trajectoires est une constante des témoignages des artistes avec lesquelles je me suis entretenue, qui disent bénéficier d'une forme de reconnaissance institutionnelle depuis quelques années seulement : « depuis is ans », dit Esther Ferrer alors qu'elle a 78 ans, c'est-à-dire depuis ses 63 ans; pour Nil Yalter, cela s'articule avec le fait d'avoir une galerie, en 2oro, c'est-à-dire l'année de ses 62 ans. La viabilité économique du travail artistique est donc arrivée pour ces deux artistes juste après leur passage de l'âge moyen de départ à la retraite.

Les modalités discursives à travers lesquelles ces deux artistes présentent la performance pendant notre entretien sont donc axées autour de la précarité, mais plus précisément autour d'une double formulation dans laquelle on peut reconnaître « la tension entre une "bonne précarité” et une "mauvaise précarité” », caractéristique du travail critique de la performance ${ }^{32}$. D'une part, le fait de ne pas pouvoir vivre de sa production artistique est une condition de travail négative dans laquelle l'artiste est vulnérable aux discriminations, en proie aux « inquiétudes » liées à l'absence de légitimité institutionnelle, et a un accès restreint au « confort matériel ». Mais le rapport de ces artistes à la précarité, c'est aussi la « grande joie » ressentie par Nil Yalter quand il n'y a rien à vendre, ou encore le malaise d'Esther Ferrer quand il faut vendre : la productivité « à perte » de la performance est aussi un outil critique vis-à-vis d'une économie artistique capitaliste basée sur la marchandisation des objets, et une source de «liberté » ou d'autonomie dans le travail. La discrétion se mue en stratégie professionnelle de la part des artistes qui mettent au centre de leur démarche ce « principe d'incertitude 33 » qui figure aujourd'hui comme l'un des principes cardinaux du travail créateur.

32 Nicholas Ridout et Rebecca Schneider, «Precarity and Performance: An Introduction », TDR: The Drama Review, vol. 56, $\mathrm{n}^{\circ} 4$, hiver 2012 , p. 8.

${ }_{33}$ Selon Pierre-Michel Menger, «L'hypothèse de départ est donc simple: le travail artistique est modelé par l'incertitude. L'activité de l'artiste chemine selon un cours incertain, 


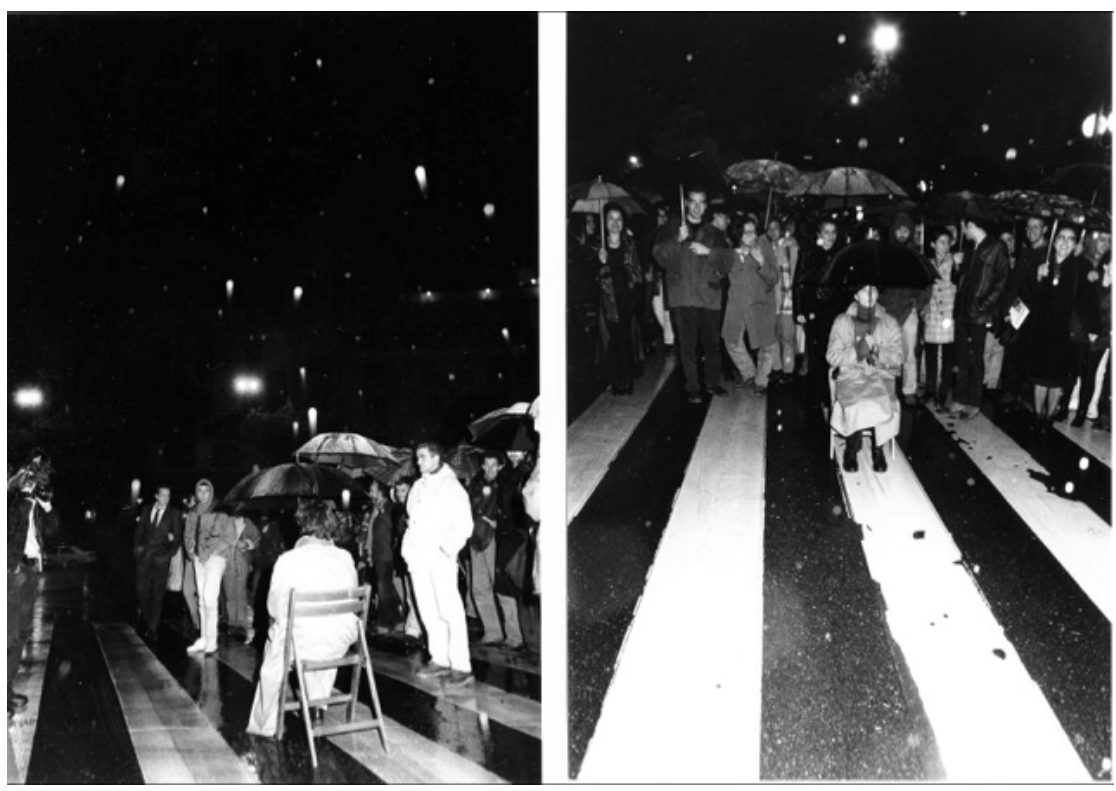

Fig. 3. Esther Ferrer, 13 acciones para 13 semáphoros (I3 actions pour 13 feux rouges), Plaza de la Cibeles, Sin número-Arte Acción, Círculo de Bellas Artes, Madrid (Espagne), 1996. Avec l'aimable autorisation de l'artiste.

\section{CONCLUSION}

To trouble temporal linearity - to suggest that time may be touched, crossed, visited or revisited, that time is transitive and flexible, that time may recur in time, that time is not one-never only one-is to court the ancient (and tired) Western anxiety over ideality and originality. The threat of

et son terme n'est ni défini ni assuré. » Pour Pierre-Michel Menger, les carrières artistiques sont caractérisées par une absence de répétition des tâches et par le fait que le.a travailleur.euse artiste ait une maîtrise limitée sur l'issue de son activité. Cela en fait des carrières « à risque ». La notion d'incertitude recouvre dans sa thèse plusieurs caractéristiques de l'activité artistique, tels l'imprévisibilité du succès, l'indéterminabilité des gains et leur écart vis-à-vis de l'effort fourni pour les atteindre; également, la faiblesse des chances d'atteindre des gains élevés et la part importante des gains non-monétaires. Cette notion permet ainsi d'expliquer à la fois le haut niveau d'attractivité des professions artistiques et les très fortes inégalités de revenus qui les caractérisent. Pierre-Michel Menger, Le travail créateur. S'accomplir dans l'incertain, Paris, Gallimard-Seuil, coll. « Hautes Études », 2009, p. 8. 
theatricality is still the threat of the imposter status of the copy, the double, the mimetic, the second, the surrogate, the feminine, or the queer ${ }^{34}$.

Le terme queer, tel que Rebecca Schneider le définit ici, désigne une esthétique intrinsèquement définie par la nécessité de la répétition, de la théâtralité et $d u$ dédoublement. Il renvoie à des modes de narrativité dans lesquels l'enchevêtrement des temporalités est nécessaire et évoque une historiographie pour laquelle la corporéité est familière, une écriture qui comprend les affects corporels comme des composants essentiels.

J'ai voulu montrer que l'entretien sur la performance en appelle précisément à ce type d'esthétique, de narration et d'historiographie : les œuvres apparaissent dans le récit à travers ces matériaux incarnés et elles demandent qu'on les écoute à partir d'une sensibilité; elles se dessinent dans la remémoration avec fragilité et discrétion, et elles impliquent des modes d'analyse qui intègrent la précarité esthétique et économique comme un axe conceptuel; elles se montrent enfin criblées de doutes, et elles requièrent de penser la faillibilité et l'omission comme des principes d'écriture à part entière. Certains de ces principes de narration diffèrent de ceux qui émanent de la documentation visuelle et scripturale, notamment la centralité des entours des œuvres et de la dimension vocale.

L'entretien peut en conclusion être envisagé comme un exercice mémoriel qui participe, comme le reenactment mais avec des moyens narratifs spécifiques, à la réécriture de l'histoire des ouvres, à la fois des pièces dans leur singularité (les œuvres), mais aussi du travail dans sa généralité (l'œuvre). Si l'objectif de cet article est de mettre en avant la spécificité de l'oralité pour la transmission des œuvres performatives, il ne s'agit pas de dire que ces deux types de traces sont incompatibles ni exclusifs. Nous avons pu observer qu'en dépit de sa forme souvent errante, le discours oral n'échappe pas à la standardisation des énoncés qui pourrait être attribuable à l'imprimé et qu'il n'exclut pas non plus la fixation visuelle issue des photographies. Plutôt que d'insister sur leur incompatibilité ou leur exclusivité, il s'agit donc ici plutôt de parier sur l'hybridation des supports mémoriels, matériels et immatériels.

\footnotetext{
34 Schneider, 20II, p. 30.
} 

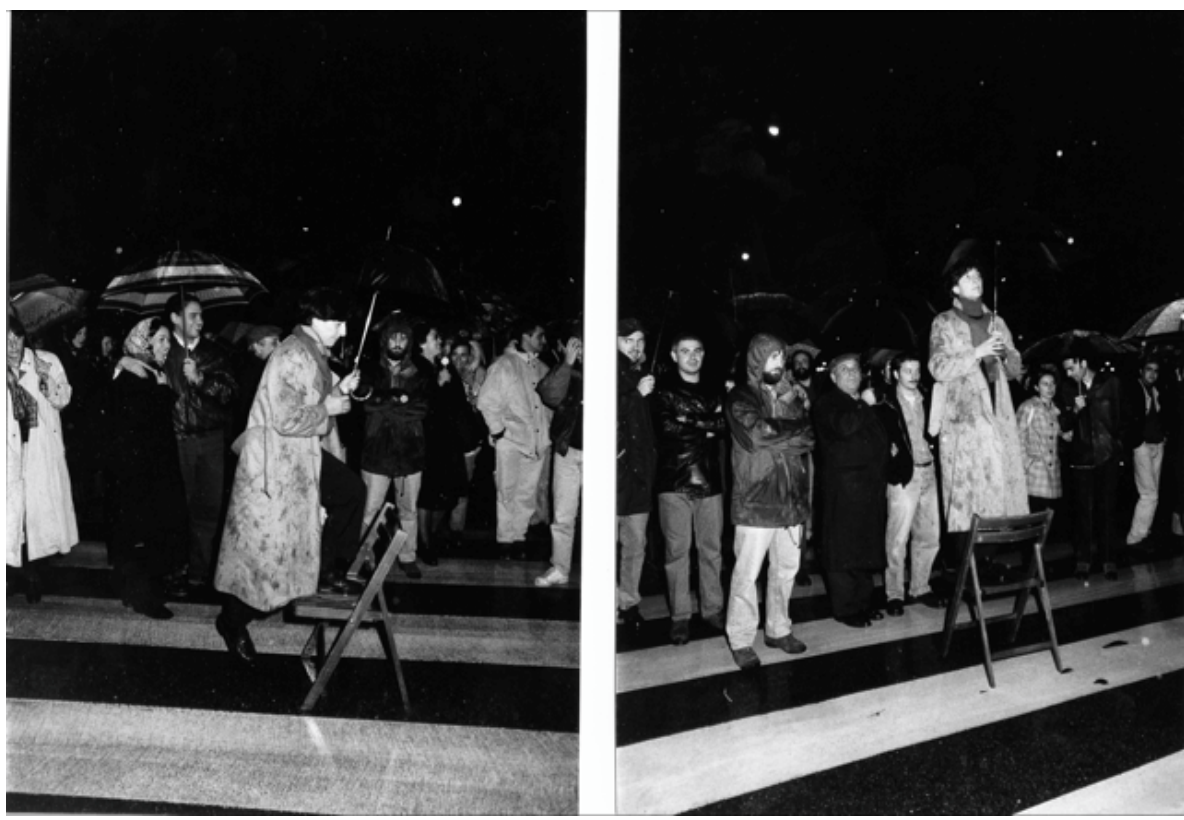

Fig. 4. Esther Ferrer, 13 acciones para I3 semáphoros (I3 actions pour 13 feux rouges), Plaza de la Cibeles, Sin número-Arte Acción, Círculo de Bellas Artes, Madrid (Espagne), 1996. Avec l'aimable autorisation de l'artiste. 13 acciones para 13 semáphoros (I3 actions pour 13 feux rouges) est une performance réalisée à Madrid lors d'un festival de performance, qui consiste en 13 brèves actions (comme monter sur une chaise), chacune réalisée devant un feu de signalisation et le temps d'un éclairage rouge, c'est-à-dire pendant l'arrêt de la circulation. On y retrouve la présence de chiffres, un certain minimalisme, et le rapport au temps très marqué qui caractérisent le travail performatif d'Esther Ferrer. 


\title{
Raconter la performance : l'entretien comme cadre pour la reprise et la transmission des performances. Entretiens avec Esther Ferrer et Nil Yalter
}

\author{
ClÉlia Barbut \\ CERLIS, Université Paris 3 SORBONne NOUVElle \\ EA Histolre ET CRitique des ARTS, Université RENNES 2
}

\section{RÉSUMÉ}

À partir d'entretiens avec les artistes contemporaines Esther Ferrer et Nil Yalter, l'auteure interroge les vertus de l'entretien pour l'histoire de l'art et en particulier pour l'histoire des œuvres performatives. Elle aborde les enjeux de l'oralité comme source et comme matière, et tente d'ouvrir des pistes de recherche historiographiques autour des affects et des incertitudes.

\section{ABSTRACT}

Based on interviews with contemporary artists Esther Ferrer and Nil Yalter, the author interrogates the value of the interview itself for art history, particularly for the history of performance art. The article focuses on the challenges of oral archives both as source and subject, and attempts to open new pathways for historiographic research into the role of affect and uncertainty.

\section{NOTE BIOGRAPHIQUE}

Chercheuse associée au CERLIS (Université Paris 3 Sorbonne Nouvelle) et à l'EA Histoire et critique des arts (Université Rennes 2), CléLIa Barbut travaille sur la performance et les pratiques performatives en histoire de l'art et en sociologie. Ses intérêts de recherche portent sur les formes narratives liées à l'oralité, les archives, le travail créateur et les épistémologies féministes. Ses publications comprennent: «Valeurs du réel et de la réalité dans l’art corporel français des années 1970 », Ownreality, n Io, 20I5; «Duchenne's Smile: Survivals and Displacements: Writing (Oneself) into the Story », dans Su Alonso et Inés Marful, RE-ACTION. Genealogy and Countercanon, catalogue d'exposition, Majorque, Casa Solleric, 2016; «Abécédaires domestiques chez Suzanne Lacy et Martha Rosler : pédagogies féministes et déraillements », Ligeia. Dossiers sur l'art, XXIXe année, n ${ }^{\circ} 53-156$, janvier-juin 2017; « Du document aux entretiens. Transmission, mémoire et historiographie des performances féministes des années 1970 », dans Émilie Blanc, Marie-Laure Allain Bonilla, Johanna Renard et 
Elvan Zabunyan(dir.), Subjectivités féministes, queer et postcoloniales en art contemporain, Rennes, Presses Universitaires de Rennes, à paraître. 